\title{
KIT DE FUGA PARA QUEDARSE- Incluye manual de fuga hacia Idilia: Herramientas para atender a la necesidad de cuidado de los cuerpos habitantes de la Facultad de Bellas Artes de la UPV/EHU situándolos en un nuevo espacio pedagógico.
}

\author{
Ione Sagasti Alegria ${ }^{a}$ e Izaskun Alvarez Gainza ${ }^{\text {b }}$ \\ aDepartamento de Dibujo UPV/EHU ione.sagasti@ehu.eus y bepartamento de Dibujo UPV/EHU izaskun.alvarezgainza@ehu.eus
}

\begin{abstract}
The Escape Kit to Stay arises as a practical response to the constitutive ailments of an increasingly fragmented academy. The fragmentation of space, time and subjects in the Faculty of Fine Arts of the University of the Basque Country, feed the paradoxes fruit of incompatible missions in the search for freedom and homogeneity. The traditional disciplinary distribution, the implementation of the Bologna Plan or the individualizing pedagogical practices, are some of the dimensions that constitute this reality. The Escape Kit consists of a Manifesto that stops in these fragmentations as a functional and experiential problem that makes a positive relationship with degree studies impossible, and a Kit with utensils to manipulate that functions as a generative metaphor and political device. People who study or work in the Faculty are invited-encouraged to make a playful and subversive use os a series of tools gathered in the kit with the aim of generating an aesthetic event that produces unexpected relationships, allowing small devices to be intra-activated with structuring mecanisms, causing phenomena that teach us other ways of thinking about our relationship with the academic environment.
\end{abstract}

Keywords: kit, academic environment, affection, academic fragmentation, functionalism-dysfunction, political device, aesthetic event.

\begin{abstract}
Resumen
El Kit de Fuga para Quedarse surge como respuesta práctica a las dolencias constitutivas de una academia cada vez más fragmentada. La fragmentación del espacio, del tiempo y de los sujetos en la Facultad de Bellas Artes de la Universidad del País Vasco, alimentan las paradojas fruto de misiones incompatibles en la búsqueda de la libertad y la homogeneidad. La tradicional distribución disciplinar, la implantación del Plan Bolonia o las prácticas pedagógicas individualizadoras, son algunas de las dimensiones que constituyen esta realidad. El kit de fuga se compone de un Manifiesto que se detiene en estas fragmentaciones como problema funcional y vivencial que imposibilita una relación positiva con los estudios de grado, y un Kit con utensilios para manipular que funciona como metáfora generativa y dispositivo político. Se invita-incita a las personas que estudian o trabajan en la facultad a hacer un uso lúdico y subversivo de una serie de herramientas reunidas en el kit con el objetivo de generar un evento estético que produzca relaciones inesperadas, posibilitando que intra-accionen pequeños dispositivos con aparatos estructurantes, provocando fenómenos que nos enseñen otras formas de pensar nuestra relación con el entorno académico.
\end{abstract}

Palabras clave: kit, entorno académico, afección, fragmentación académica, funcionalismo-disfunción, dispositivo político, evento estético. 


\section{Introducción}

Hay una opinión generalizada de que la Facultad de Bellas Artes de la UPV/EHU no es un edificio amable, de que sus espacios no son acogedores: hace frío o calor, el aire no circula, la luz predominante es fluorescente y te envuelve como un laberinto de altos muros que elevan las escasas ventanas sobre nuestras cabezas en los despachos y las ocultan en recovecos del techo al llegar a las aulas. Se organiza sobre una retícula de estancias estancas rectilíneas que imposibilitan el contacto corporal y visual.

Esta disposición tiene sus causas y efectos. La forma viene determinada por la división académica del saber en ramas y niveles -disciplinas y progreso-, y por la idea de que el saber está guardado a buen recaudo en su interior. Y ahora esta forma es alimentada por la fragmentación y compresión del tiempo con la llegada de los nuevos planes docentes que empuja hacia el funcionalismo educativo y la disfunción. Todo ello afecta directamente a las dinámicas vitales del espacio académico, provocando simulacros de acuerdos y violencias sistémicas que derivan en desconexión y desafección. El clientelismo y la maquinaria de promoción académica quedan compartimentadas en espacios virtuales y físicos acotados que no dejan ver, sentir e imaginar otras formas de ser y relacionarse.

Habiendo recogido en el marco de una investigación sobre la política de los afectos en las relaciones pedagógicas en la Facultad de Bellas Artes ${ }^{1}$ información sobre las afecciones del habitar sus espacios, el Kit de fuga para quedarse surge como respuesta práctica a la detección de los peligros de permanecer en ellos y la necesidad del cuidado de los cuerpos y su ambiente. Como solución hemos iniciado un proceso de fuga, una respuesta declarativa y radical que posibilite desplazar los sujetos hacia otro lugar aún por construir y que ofrezca una visión activa y afirmativa de la conquista y defensa de un nuevo territorio pedagógico. Se huye para quedarse, queremos quedarnos, y por ello el plan se desarrolla dentro del sistema, tendiendo la mano a otros, ideando un kit básico de usuario con sus útiles y un manual.

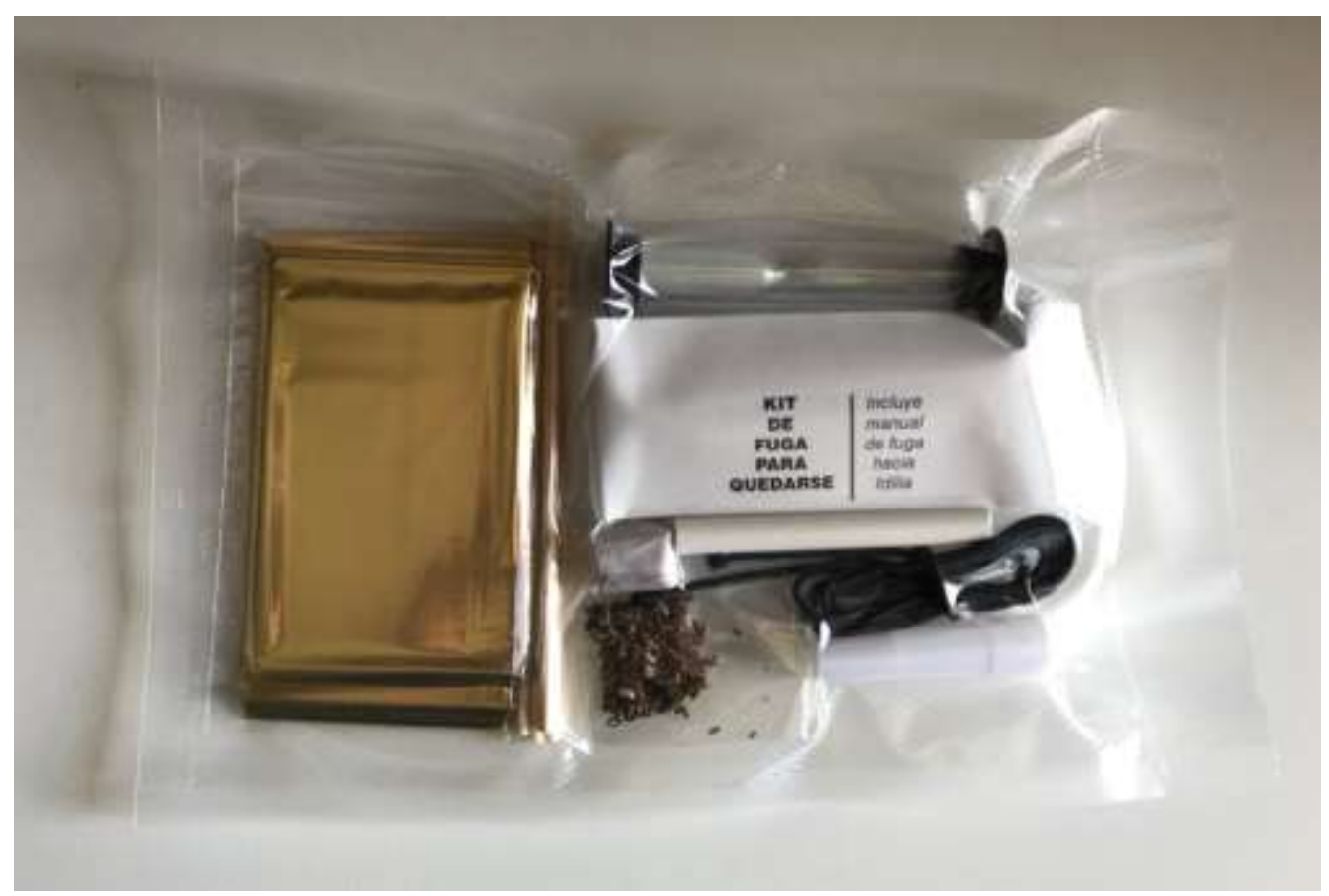

Fig. 1 Kit de Fuga para quedarse

\footnotetext{
${ }^{1}$ Investigación en curso. "La política de los afectos en las relaciones pedagógicas en la enseñanza del arte: un estudio de caso. Cómo la política de los afectos transitan y repercuten en la producción de conocimiento y experiencia". Programa de doctorado Investigación y Creación en Arte. UPV/EHU.
} 


\section{Fragmentaciones académicas}

A lo largo del curso académico 2018-2019 se diseñaron y entregaron unas "invitaciones de tesis" para que profesores, alumnos, y personal de administración y servicios pudieran ayudarme a detectar unas líneas de trabajo en relación a la vida de los afectos en la facultad, qué nos toca o tocamos, y cómo nos afecta ${ }^{2}$. Se les pedía una pregunta para confeccionar una posible encuesta y una aportación libre. Recogidas las aportaciones de los invitados-participantes, se realizó su clasificación y detección de las dimensiones afectivas que pudieran ser de interés para el desarrollo de la investigación. Agruparse, la exclusión, los muros, la falta de tiempo, los ritmos de trabajo, la falta de luz o la hora de la comida son algunas de los agentes o factores que surgieron, que me llevaron a pensar de forma más generalizada en las formas de fragmentación que hay (temporal-espacial-sujetos) y las formas de violencias (democráticas, afectivas, ambientales).

Los estudios superiores de arte en la UPV/EHU se organizan distribuidos en ramas de progresión por niveles y disciplinas que se imparten en aulas específicas agrupadas en pasillos asignados al área. A esta división del saber en áreas de conocimiento, y la escisión filosófica del saber científico frente al sentir artístico como formas de abordar la realidad, se ha sumado en los últimos años la fragmentación del tiempo con la llegada del Plan Bolonia. Esta fragmentación múltiple ha marcado la estructura e infraestructuras de las instituciones académicas parcelando el espacio en estancias estancas que acogen sesiones lectivas cada vez más breves, condicionando ritmos y formas de proceder. Se han perdido espacios físicos y temporales para trabajar. Los tiempos de éste modelo común son otros, se han fragmentado y acortado provocando de reflejo que los espacios de trabajo se compriman hasta desaparecer, reduciendo nuestra parcela ocupable a las taquillas o mesas de despacho. Esto hace que la vida agonice en la facultad.

Además, las formas, como las palabras, codifican nuestros modos de sentir y pensar, las formas arquitectónicas por ello son condiciones que afectan a nuestra manera de movernos y ser. La facultad de Bellas Artes tiene unos elementos arquitectónicos muy característicos, entre ellos las paredes de bloques de hormigón visto que separan los espacios. Estos grandes ladrillos se hacen aún más presentes debido a la ausencia de ventanas en las paredes de las aulas y al superar la altura de las cabezas en los despachos. No vemos lo que ocurre en otros espacios o en el exterior, haciendo que perdamos la noción del tiempo y la comunicación, convirtiendo en guetos algunos lugares, dejando otros olvidados, enfatizando su fragmentación disciplinar estructural.

Siguiendo la idea de Hito Steyerl (2010) de que una disciplina es una forma de opresión que indica un conflicto reprimido, esquivado o potencial, observamos que nuestra Facultad puede esconder muchas violencias sistémicas que podemos detectar ya desde su arquitectura. En la instalación “The Building” (2009) Steyerl realiza una intervención en la Academia de Arte de Linz -edificio construido por presos durante el nazismo, en un acto revelador para mostrar cómo los espacios de la Academia pueden revelar la disciplina que clasifica y nombra, los rastros de su vida y sus conflictos, en este caso, a través de mapas simbólicos que rastrean sus historias de terror. Así que el edificio se convierte ahora en el instrumento para detectar sus propias fuerzas de constitución e institucionalización.

Para el arquitecto Juhani Pallasmaa, "desde el punto de vista intelectual, podemos haber rechazado la dualidad cartesiana de cuerpo y mente desde un punto de vista filosófico, pero la separación sigue vigente en las prácticas culturales, educativas y sociales" (Pallasmaa 2012, 9). El arquitecto nos invita a atender los sentidos y buscar lo habitable.

Además de la fragmentación espacial y temporal, también se produce una fragmentación de los sujetos debido a procesos individualizadores que esculpen creadores aislados, en un individualismo colectivo que nos aleja de la coimplicación que defiende Marina Garcés en Un mundo común. Garcés nos habla de la imposibilidad de ser sólo un individuo $(2013,49)$ en detrimento de un Nosotros que se construye como continuidad de los cuerpos y no como la suma de individuos. Esta coimplicación sería la que permite poner en marcha de manera colectiva procesos de transformación de aquello que nos afecta. Estas tres dimensiones fragmentadas (tiempo-espacio-sujeto) interfieren, entre otras cosas, en la creación de una comunicación fluida, acotándola en reuniones o sesiones separadas por áreas y programadas desde lejos, que derivan en tensiones y disfunciones, provocando una desafección por las dinámicas generales del centro o la institución. 2 La investigación se plantea como una investigación basada en la práctica inspirada por el Giro Afectivo en Ciencias Sociales e interesada por una ontoepistemología
nuevo materialista y las teorías "post" (posthumanismo, postcualitativa, postestructuralismo). 


\section{Incomodidades democráticas y problematizar lo consensuado}

En la educación artística superior nos enfrentamos a dos tensiones de fondo que hacen chirriar sus postulados generando contradicciones y confrontaciones. Por un lado, está la tensión del deseo de formar en el pensamiento crítico y libre desde una estructura académica concebida en base a una lógica disciplinar. Por otro lado, está la tensión de estar enseñando métodos y metodologías para la práctica artística -que se han entendido históricamente como contenedoras de un germen transgresor o subversivo-, desde la misma estructura disciplinar que fragmenta, generaliza y normaliza los saberes. Una uniformización de prácticas y conductas que limita o esconde las diferencias fruto de un consenso que excluye aquello que no encaja o no rema en la misma dirección. Hay una coexistencia incómoda, o como lo expone Vidal (2013) físicamente imposible entre estos dos universos paralelos que son la homogeneidad y la diversidad.

Para salvar estas y otras incomodidades y violencias democráticas que niegan, apartan e ignoran unas cosas a favor del consenso, Chantal Mouffe defiende una política democrática que contemple la controversia inherente a la democracia siempre en tensión con la lógica del liberalismo. Para ello propone una democracia agonística (de adversarios y no de enemigos) donde convivan las diferencias y se mantenga viva la controversia consustancial a la democracia (Mouffe 2007, 10).

Además de contemplar el mutuo disenso como posibilidad democrática, Rancière va más allá al proponer que sea problematizando lo consensuado - a través del conflicto y la negociación surgidas en el disentimiento-, lo que nos posibilite acceder a un nuevo conocimiento que no reproduzca lo sabido. El consenso "significa propiamente un modo de estructuración simbólica de la comunidad que evacúa lo que constituye el corazón de la política, es decir, el disenso" (Rancière 2012, 108).

Con la idea de lograr un nuevo conocimiento, Irit Rogoff se propone producir un conocimiento no enmarcado, "unframed knowledge", por órdenes disciplinarias o temáticas, sino un conocimiento que se presente en relación a una urgencia, "urgent issue", en relación a las presiones y dificultades de la contemporaneidad. Rogoff argumenta en la publicación especial "Education Actualized" de e-flux journal cómo buscaba salirse de los convencionalismos en torno al conocimiento al plantear que se contemplase la educación gratuita en su universidad. Libre de convenciones, el conocimiento no enmarcado, nos argumenta, está menos arraigado genealógicamente y puede avanzar en vez de retroceder (Rogoff 2010, 40). Rogoff genera una disrupción en los procesos de pensamiento habituales en su universidad, como docente parte de la institución propone desde dentro del sistema una cuestión que genera un evento que subvierte el orden consensuado y normalizado.

\section{Un manifiesto y un kit}

Como respuesta a las dolencias detectadas en la vida académica de nuestra facultad, hemos ideado un Kit de Fuga para Quedarse. Queremos quedarnos en la facultad pero para ello vemos la urgencia de alterar la realidad desde tres ámbitos (espacio-tiempo-sujeto). El kit de fuga se compone de un Manifiesto que se detiene en la fragmentación espacial y temporal como problema funcional y vivencial que imposibilita una relación positiva con los estudios de grado en la Facultad de Bellas Artes, y un Kit con utensilios para manipular que funciona como metáfora generativa y dispositivo político.

En el manifiesto, primero se expone la situación que se está viviendo, y después se apunta la posibilidad de crear cambios para devolver la vida a la facultad, y lograr que nos quedemos más tiempo en ella habitándola de otra manera. Se invitaincita a las personas que estudian o trabajan en la facultad, a hacer un uso lúdico y subversivo de una serie de herramientas reunidas en el kit con el objetivo de generar un evento estético que produzca relaciones inesperadas, posibilitando que intra-accionen pequeños dispositivos con aparatos estructurantes, provocando fenómenos que nos enseñen otras formas de pensar nuestra relación con el entorno académico.

Es un arma de resistencia portátil: compacto, ligero y envasado al vacío, listo para abrir y activar cuando se necesite/desee alterar el entramado sociomaterial. El envasado al vacío nos remite a la conservación en el tiempo, es algo que te espera, espera a que estés preparado para consumirlo, y a los viajes largos hacia terrenos desconocidos. Con la idea de lo portátil 
que puedes llevar en el bolsillo y desplegar para su uso, Martín Azúa propone la Casa Básica ${ }^{3}$, un volumen habitable de prestaciones básicas; plegable, hinchable, reversible, que utilizando la más avanzada tecnología propone una casa casi inmaterial para responder a la necesidad en nuestra sociedad de entender el hábitat de una manera más esencial y razonable.

Encontramos otros ejemplos de obras portátiles para ser manipuladas y activadas en situaciones de necesidad en el formato kit en Fluxus. Con una actitud lúdica y mucha ironía, estás pequeñas cajas interactivas se tornan radicales por su urgencia latente y al convertir al espectador en su usuario interpelado a alterar algún aspecto de su realidad.

Nuestro Kit de Fuga es una rebeldía de juguete que consideramos site y time specific ya que lo entregamos a principios de curso esperando que sus usuarios lo activen a lo largo del mismo y poder recoger así las experiencias a través del correo electrónico y la plataforma Instagram, entre otros medios. Nos interesa detenernos en la dimensión temporal de los acontecimientos que se puedan generar para pensar según la lógica de Rancière, que el sujeto político surge como "estallido", sólo aparece en el momento de la irrupción y del disenso, dando lugar a "escenas de enunciación y de manifestación que pleitean hasta con los datos sensibles de la comunidad" (Rancière en Capasso 2018, 221). Rancière "hace énfasis en la dimensión de la temporalidad, en tanto no siempre hay política, y cuando la hay, ella instaura otro curso del tiempo. Es decir, los momentos políticos son microacontecimientos de disenso, de desgarro del tejido común, un cuestionamiento al mundo dado y la posibilidad de configurar otro. Consideramos que esta idea de momento es productiva en la medida en que permite dar cuenta que es una instancia en la cual el carácter contingente de lo social emerge" (Capasso 2018, 221).

En un equilibrio entre lo inofensivo y lo ofensivo, el kit propone una involucración cómplice de algo inocente que juega a ser ilegal, irreverente y provocativo, para actuar en la contingencia de un momento académico. Está preparado y dispuesto para ese potencial momento catalizador.

El Kit se compone de 7 movimientos con sus dispositivos correspondientes en los que el sujeto actúa y altera el entorno revirtiendo cambios en él y su realidad. Se trata de escapar de los marcos establecidos para pensar otros. Abierto el vacío del kit, queda abierta la potencia de actuar sobre tres dimensiones del ámbito académico: ESPACIO, SUJETO Y TIEMPO, posibilitando ver en el reverso la trenza que los relaciona. Abrir, ocupar, cultivar, construir, cambiar, renegar y parar, son los estadios de un plan de acción que se propone una transformación de la realidad a todos los niveles, desde el ecosistema, pasando por los cuerpos hasta llegar a transgredir las leyes físicas del mundo conocido. Alterar- intervenir EL ECOSISTEMA (FUERA); Alterar-avivar LOS CUERPOS (DENTRO), y Alterar-invertir LA DIMENSIÓN ESPACIOTEMPORAL (MÁS ALLÁ). Una TIZA para ABRIR; unas ESTAQUITAS Y GOMA para OCUPAR; unas SEMILLAS para CULTIVAR; una MANTA ISOTÉRMICA para CONTRUIR; un CARAMELO para CAMBIAR; un MATASUEGRAS para RENEGAR; y un RELOJ DE ARENA para PARAR. Cada artículo tiene en las Instrucciones de Uso del Manual unas indicaciones que apuntan hacia donde pueden dirigirse para accionar algún aspecto de la realidad.

3 La "Casa Básica" de Martín Azúa, 1999. Forma parte de la colección permanente del MOMA de New York desde 2007. https://www.martinazua.com/es/producto/casa$\underline{\text { basic }}$ 


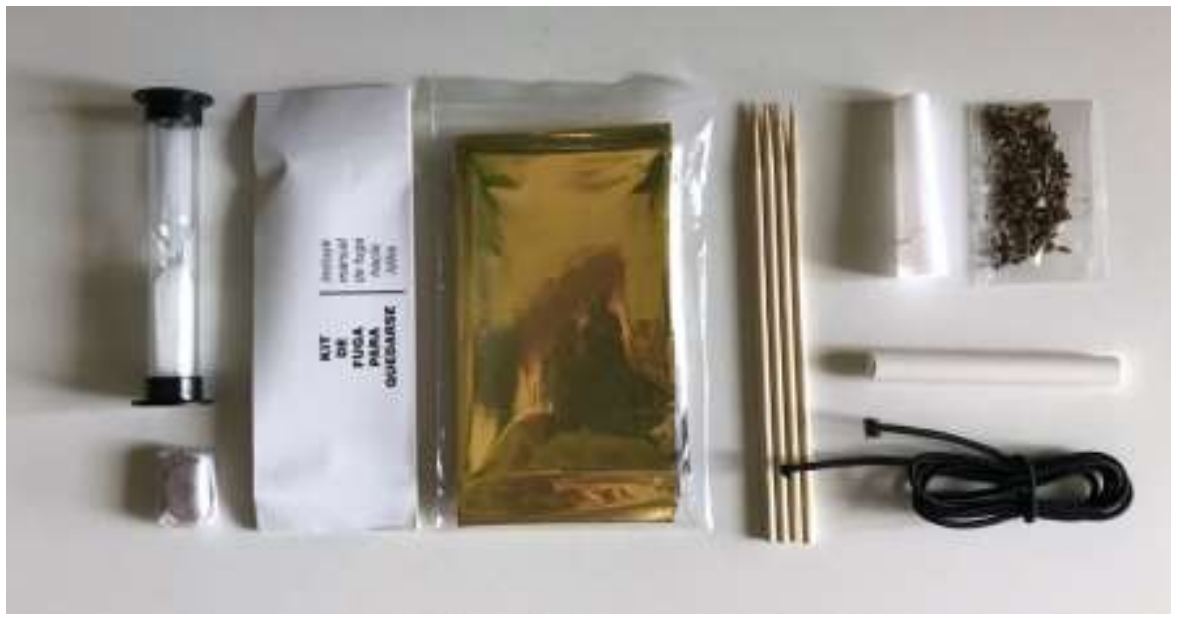

Fig. 2 Elementos del Kit de Fuga

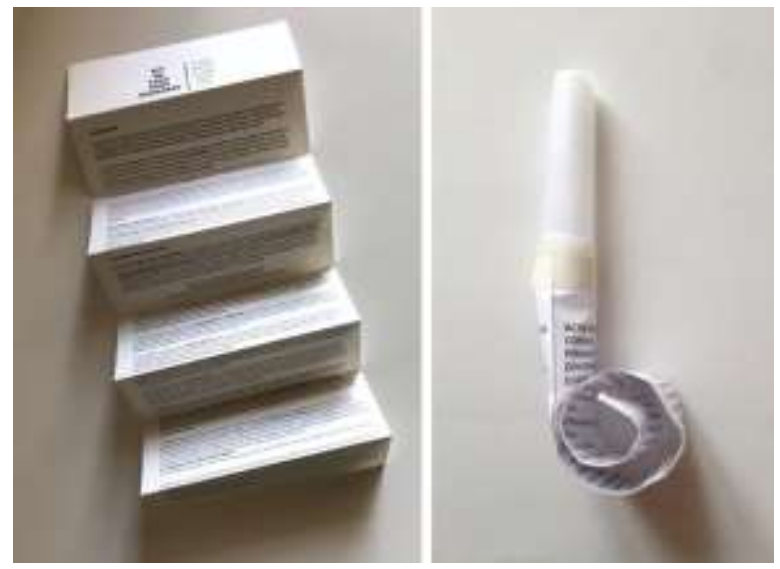

Fig. 3 Pliego con manifiesto e instrucciones de uso. Y Fig. 4 Acta matasuegras armado.

\section{Conclusiones}

$\mathrm{Al}$ idear el Kit de fuga entendemos, como Rancière, que lo político del arte no depende de la temática de la obra sino de los procedimientos poéticos y del poder de reconfigurar el reparto de lo sensible, de hacer visible lo que no lo era, en una nueva configuración de sujetos, objetos, espacios y tiempos.

Acompañan a este fin político de lo estético, la fuerza de la experiencia de lo lúdico y el poder de la emergencia, de algo que surge en el presente inmediato respondiendo a una necesidad.

El kit responde a las paradojas de la academia -causa o efecto de sus fragmentaciones y consensos-, con la capacidad política del arte de introducir lo inesperado, generando eventos estéticos que posibilitan alterar las maneras de ver, sentir y pensar la realidad.

\section{Referencias}

CAPAsso, V. (2018). Lo político en el arte. Un aporte desde la teoría de Jacques Rancière. Estudios de Filosofía, 58, pp. $215-235$.

GARCÉs, M. (2013). Un mundo común. Barcelona: Ediciones Bellaterra.

MoufFe, C. (2007). Prácticas artísticas y democracia agonística. Barcelona: Universitat Autónoma de Barcelona y MACBA.

PallasmaA, J. (2016). Habitar. Barcelona: Gustavo Gili.

PallasmaA, J. (2012). La mano que piensa. Sabiduría existencial y corporal en la arquitectura. Barcelona: Gustavo Gili.

RANCIÈRE, J. (2010). Momentos Políticos. Buenos Aires: Capital Intelectual.

RANCIÈRE, J. (2012). "El giro de la estética y de la política”. El malestar en la estética. Madrid: Clave intelectual. pp.133-161. 
RoGoFF, I. (2010). “Free”. E-flux, Journal 14. March. https://www.e-flux.com/journal/14/61311/free/

Rogoff, I. (2010). "Practicing Research / Singularising Knowledge". Mahkuzine. Journal of Artistic Research, 9: Agonistic Academies, 69-74.

Slager, H. (2012). The Pleasure of Research. Helsinki: Finnish Academy of Fine Arts.

STEYeRL, H. (2010) “Aesthetics of resistance”. Mahkuzine. Journal of Artistic Research, 8. 31-37.

VidAL, J. (2013). “La universidad informal”. Cuaderno de trabajo 2. Studia XXI. Fundación Europea Sociedad y Educación. http://www.studiaxxi.com/site/wp-content/uploads/Cuaderno-de-trabajo-2.pdf 